\title{
Nicht und kein-. Zu Auswahl und Verwendung der Negationselemente
}

1. Die Entscheidung zwischen nicht und keinist im DaF-Unterricht als fehlerträchtig bekannt. In den Grammatiken findet man dazu in unterschiedlichen Varianten die Regel, keinmüsse verwendet werden, wenn im nicht-verneinten Satz der unbestimmte Artikel oder der sogenannte Nullartikel steht. ${ }^{1}$ Diese Regel ist nicht falsch, erklärt aber wenig und versagt in der Praxis oft. Sie wird darum gewöhnlich durch mehr oder weniger lange Listen von Beispielen und Ausnahmen ergänzt. ${ }^{2}$

Den Unterschied zwischen nicht und keinversteht man am besten, wenn man davon ausgeht, daß kein- in erster Linie Quantor, nicht dagegen genuines Negationselement ist. Die Schwierigkeiten lassen sich dann darauf zurückführen, daß kein- neben seiner Quantifikationsfunktion noch andere Funktionen erfüllt. Seine Ambivalenz steht in engem Zusammenhang mit der seines positiven Gegenstücks ein-, das einerseits ebenfalls als Quantor, andererseits als Indefinitartikel fungiert.

Es ist sinnvoll, mit einigen konzeptionellen Vorüberlegungen zu beginnen. Wenn wir kommunizieren, so verständigen wir uns über Sachverhalte und Gegenstände. Ein Kommunikationsereignis besteht darin, daß ein Sender eine innere Vorstellung von einem Sachverhalt mittels sprachlicher Zeichen kodiert, die ein Empfänger aufnimmt und als Anweisungen interpretiert, seinerseits eine innere Vorstellung von einem Sachverhalt aufzubauen. Die inneren Vorstellungen kann man etwas technischer auch als Repräsentationen bezeichnen. ${ }^{3}$ Ebenso wie Sprachzeichen aus unterschiedlichen Bausteinen (Wörtern) zusammengesetzt werden, müssen auch die inneren Repräsentationen von Sachverhalten aus Bausteinen zusammengesetzt werden, nämlich unter anderem aus Repräsentationen von Gegenständen.

Jeder, der an einem Kommunikationsereignis teilnimmt, verfügt bereits über einen Vorrat an Wissen, der aus allgemeiner Lebenserfahrung und insbesondere aus früheren Kommunikationsereignissen stammt. Solches Wissen führt u.a. dazu, daß ein Empfänger den Äußerungen eines Senders mit Erwartungen begegnet, und zwar erwartet er sowohl die Verwendung bestimmter Zeichen (z. B. seiner Muttersprache) als auch Anweisungen hinsichtlich bestimmter Gegenstände und Sachverhalte (z. B. solcher, wie er sie aus seiner Erfahrung schon kennt). Derartige Erwartungen sind ein wichtiger, wenn auch nicht der einzige Anlaß, in der Kommunikation etwas zu negieren, wenn man nämlich mitteilen will, daß eine geäußerte oder vermutbare Erwartung nicht erfüllt wird. ${ }^{4}$

2. Betrachten wir zunächst das Element nicht. Es ist das wichtigste und vielseitigste Negationszeichen im Gegenwartsdeutschen.

Wenn wir etwas negieren, so zielen wir i.a. auf die Beschreibung eines Sachverhaltes, von der wir sagen, daß sie zutrifft oder nicht zutrifft, d.h. mit unserem Bild von der Welt verträglich ist oder nicht:

(1.a) Es regnet.

(1.b) Es regnet nicht.

(2.a) Johannes fegt die Veranda.

(2.b) Johannes fegt die Veranda nicht.

Negationen wie in (1.b) und (2.b) sind unbeschränkt, d.h., es wird nichts weiter gesagt, als daß die jeweilige Sachverhaltsbeschreibung nicht zutrifft.

Nun können wir, wenn wir eine Sachverhaltsbeschreibung negieren, dabei auch besonders auf einen bestimmten Bestandteil der Beschreibung zielen, den man unserer Meinung nach austauschen müßte, um die Beschreibung zu retten:

(2.c) Johannes fegt / NICHT die VeRAN $\backslash$ da. (sondern z. B. den FLUR)

(3) Monika hilft/NICHT ihrem VA \ter. (sondern z. B. der $\mathrm{O} \backslash \mathrm{ma})^{5}$

Derartige Negationen sind zum einen zugespitzt, d.h. auf ein bestimmtes Element der Beschreibung als Negationsziel gerichtet, zum anderen replaziv, ${ }^{6}$ d. h., sie fordern zur Ersetzung des Negationsziels auf. Als Nega- 
tionsziel kommt u.a. das Verb als Bezeichnung des Sachverhaltstyps in Frage:

(4) Sandra / NIMMT das Buch NICHT $\backslash$. (sondern legt es z. B. WEG)

Ferner kann Negationsziel die Beschreibung eines sachverhaltsbeteiligten Gegenstandes sein. Zur Beschreibung eines Gegenstandes können drei Arten von Informationen kodiert werden: erstens was für ein Gegenstand gemeint ist (Gegenstandskategorie), zweitens welche Menge (Gegenstandsquantität) und drittens welches Individuum (Gegenstandsidentität). In den Beispielen (2.c) und (3) werden die betreffenden Gegenstandsbeschreibungen hinsichtlich dieser Teilinformationen wiederum unbeschränkt negiert. Man kann eine Negation aber bei Bedarf auf jede der drei Beschreibungskomponenten zuspitzen:

(5) Ein Casa/NOva ist der NICHT $\backslash$. (sondern z. B. ein HAM $\backslash$ pelmann) (Gegenstandskategorie)

(6) Ich brauche / NICHT FÜNF $\backslash$ Karten. (sondern 2. B. VIER) (Gegenstandsquantität)

(7) Wir nehmen / NICHT DIE \sen Bus. (sondern z. B. JE\nen) (Gegenstandsidentität)

In replaziver Verwendung erhält das Element nicht einen erhöhten Nachdruck, was man vor allem daran erkennt, daß es, wenn keine Beschreibungsalternative explizit gemacht wird (wie in (8.b)), obligatorisch Akzentträger ist:

(8.a) Sie hat nicht Ge/BURTStag, sondern NA $\mid$ menstag.

(8.b) Sie hat / NICHT GeBURTS \tag.

3. Wenden wir uns nun kein- zu. Wenn wir dieses Element als Quantor betrachten, so kann es nicht dazu dienen, Beschreibungen von Sachverhalten hinsichtlich ihres Zutreffens oder Nicht-Zutreffens zu kennzeichnen, sondern es muß ein Mittel der Beschreibung selbst sein, und zwar ein Mittel zur Beschreibung von Gegenständen. Die Menge, die wir mit kein- angeben können, ist offensichtlich die Nullmenge. Es wäre allerdings falsch, daraus zu folgern, daß Ausdrücke wie kein Baum, kein Buch, kein Auto alle dasselbe bedeuten müßten. ${ }^{7}$

Wir haben gesehen, daß bei der Beschreibung von Gegenständen Kategorialinformation, Quantitätsinformation und Identitätsinformation zusammenkommen. Auf jede der drei Informationsarten können sich aber getrennt die Erwartungen eines Empfängers richten. Wenn wir also sagen kein Baum, so könnte man unsere Botschaft etwa ausbuchstabieren als: „Du er- wartest möglicherweise einen Baum, aber es ist keiner da." Entsprechendes gilt für kein Buch. kein Auto usw. Von daher erklärt es sich auch, warum kein- einen Plural bildet. Sagen wir keine Bäume, so lautet die Botschaft: „Du erwartest möglicherweise Bäume, aber es sind keine da." Diese ist von der Botschaft, die wir mit kein Baum übermitteln, deutlich verschieden.

Nullquantifikation ist zwar nicht das gleiche wie Negation, kann aber im Effekt auf etwas Ähnliches herauskommen. Negation sagt, daß eine bestimmte Sachverhaltsbeschreibung ganz oder in Teilen nicht mit unserem Bild von der Welt verträglich ist. Nullquantifikation sagt, $\mathrm{da} B$ in einer bestimmten durch einen Sachverhalt definierten funktionalen Position, wo etwas sein könnte oder sollte, nichts von dem, was dort sein könnte oder sollte, wirklich ist. Äußere ich etwa:

(9.a) Ich habe keine Wohnung gefunden.,

so sage ich damit zunächst einmal nur, daß unter den Dingen, die ich möglicherweise gefunden habe, keine Wohnung ist, und erst als Ergebnis weitergehender Interpretation auf der Empfängerseite ergibt sich, daß demgemäß eine Beschreibung wie:

(9.b) Ich habe eine Wohnung gefunden.

unzutreffend sein müßte.

In diesem Zusammenhang ist es nützlich, die konzeptionellen Überlegungen zum Sprechen über Gegenstände noch etwas zu vertiefen. Informationen über die Gegenstandskategorie werden typischerweise mit Hilfe von Substantiven und Adjektiven kodiert. Man kann solche Elemente auch als Deskriptoren bezeichnen:

(10) Elke trägt eine grüne Schleife.

Mit der Beschreibung der Gegenstandskategorie ist in den meisten Fällen bereits klar, ob der Gegenstand gegliedert (in Individuen) oder ungegliedert (als Masse) vorliegt. Dieser Unterschied wirkt sich auf die Möglichkeit aus, die Gegenstandsquantität zu bestimmen, denn gegliederte Gegenstände werden gezählt, ungegliederte dagegen gemessen:

(11.a) Monika kaufte zwei Brötchen und zwei Liter Milch.

Quantitätsinformation kann grammatisch über den Numerus und lexikalisch über Quantoren kodiert werden:

(12.a) Puffi benutzt ein Wörterbuch.

(12.b) Puffi benutzt mehrere Wörterbücher. 
Die Informativität des Numerus ist im Deutschen auf die Frage beschränkt, ob eine Mehrzahl von Individuen (Plural) vorliegt oder nicht. Für eine Einzahl von Individuen und eine zu messende, ungegliederte Menge wird gleichermaßen der Singular verwendet. Der hier auftretende Unterscheidungsbedarf muß durch Quantoren gedeckt werden. Für eine Einzahl von Individuen steht der Quantor ein- zur Verfügung; für die quantitative Beschreibung einer ungegliederten Menge verwenden wir Elemente wie ein bißchen, wenig, viel usw. Bei ungegliederten Mengen sind Quantifikationselemente grundsätzlich fakultativ. Der Singular allein leistet hier offenbar schon eine minimale Quantifikation, die etwa ausbuchstabiert werden könnte als „eine bestimmte Quantität“:

(11.b) Monika kaufte Milch.

Bei individuellen Gegenständen ist das Quantifikationselement ein-obligatorisch:

(12.a) Puffi benutzt ein Wörterbuch.

(12.c) *Puffi benutzt Wörterbuch.

Allerdings scheint es, unter Informativitätsgesichtspunkten betrachtet, in vielen Fällen weitgehend redundant. Wörterbücher beispielsweise liegen definitionsgemäß in Form von Individuen vor, so da $B$ der Singular hier ohnedies nur als Bestimmung der Einzahl verstanden werden kann. Es dürfte diese Redundanz sein, die die Interpretation von ein- als Indefinitartikel fördert.

Auch eine Mehrzahl von Individuen kann bei Bedarf durch Quantoren genauer bestimmt werden. Hierzu dienen Elemente wie die Zahlwörter $z w e i$, drei, vier usw., ferner ein paar, einige, eine Reihe usw. Da der Plural aber schon an sich eine minimale Bestimmung der Gegenstandsquantität leistet (auszubuchstabieren etwa als „mehr als ein-“), sind diese Elemente fakultativ:

(11.c) Monika kaufte Brötchen.

Bei allen quantifizierten Gegenstandsbeschreibungen bestehen nun zwei Möglichkeiten ${ }^{8}$ : Entweder es geht um irgendwelche Gegenstände der betreffenden Kategorie, deren Identität nicht festliegt, oder der Sender hat ein bestimmtes Individuum bzw. eine bestimmte Gruppe oder Menge im Auge. Im ersten Fall spricht man in bezug auf die Dekodierung von der unspezifischen Lesart der Quantifikationselemente:

(13) Ich brauche eine neue Glühbirne. ( $\rightarrow$ irgendeine neue Glühbirne), im zweiten von der spezifischen Lesart:

(14.a) Peter beobachtete zwei Männer. ( $\rightarrow$ zwei bestimmte Männer)

In bezug auf die Vorstellung des Senders kann man auch von variablen und fixierten Gegenständen sprechen.

Quantoren werden unspezifisch verwendet, wenn die Identität des betreffenden Gegenstandes nicht wichtig oder nicht klar ist. In diesem Fall steht ihre quantifizierende Funktion im Vordergrund. Sie werden spezifisch verwendet, wenn der Sender die Identität des betreffenden Gegenstandes in die Kommunikation einführen möchte. In diesem Fall steht ihre Funktion als Indefinitartikel im Vordergrund. An Gegenstandsbeschreibungen mit spezifisch verwendeten Quantoren wird typischerweise mittels anaphorischer Elemente (Demonstrativelemente, z. B. Definitartikel, oder Pronomen der dritten Person) angeknüpft:

(14.b) Peter beobachtete mit Interesse zwei Männer. Diese waren dabei, einen Mercedes zu stehlen.

Ist ein Gegenstand in die Kommunikation eingeführt, so muß weder die Gegenstandskategorie noch seine Quantität weiterhin bestimmt werden. Es genügt dann, durch Anaphern klarzumachen, welcher Gegenstand an einer bestimmten Stelle gemeint ist:

(15) Es waren einmal ein König und eine Königin. Er liebte schnelle Autos und sie den Kammerdiener.

Bei Bedarf kann aber durchaus die Beschreibung der Kategorie wiederaufgenommen werden:

(16) Es war einmal ein Mann, der hatte einen Schwamm. Der Schwamm war ihm zu naß, da ging er auf die Gaß. (...)

Auch die Bestimmung der Quantität kann wiederholt werden:

(17) Am Straßenrand saßen zwei Kinder und froren. Vielleicht waren die zwei von zu Haus weggelaufen.

Während Bestimmungen der Kategorie und der Quantität bei eingeführten Gegenständen fakultativ sind, ist die Bestimmung der Identität durch Pronomen oder Artikel obligatorisch. Ich werde die dazu verwendbaren Elemente, leicht abweichend von der üblichen Terminologie, zusammenfassend als Determinatoren bezeichnen.

Wir können somit drei Varianten des Sprechens über Gegenstände unterscheiden:

(i) variabler Gegenstand:

(Quantor +) Deskriptor + Numerus 
(ii) einzuführender fixierter Gegenstand: (Indefinitartikel + ) Deskriptor + Numerus

(iii) eingeführter fixierter Gegenstand:

Determinator (+ Quantor) (+ Deskriptor) + Numerus

Die Verwendung von kein- ist zunächst einmal für die erste dieser Varianten typisch, bei der Quantifikation besonders wichtig ist. Nullquantifikation kann dabei mit beiden Numeri sowie mit Bezeichnungen für gegliederte und ungegliederte Gegenstände verbunden werden:

(18.a) Monika kaufte keine Bröıchen.

(19.a) Monika kaufte keine Milch.

(20.a) Monika kaufte keine Glühbirne.

Darüber hinaus sind bei Bedarf auch Kombinationen mit anderen Quantoren möglich:

(21) A Hast du hier zwei merkwürdig gekleidete Männer gesehen?

B Ich habe keine zwei Männer gesehen.

Nun kann man Sätze wie (18.a) bis (20.a) auch so interpretieren, daß sie das Nicht-Zutreffen von Sachverhaltsbeschreibungen wie (18.b) bis (20.b) einschließen:

(18.b) Monika kaufte Brötchen.

(19.b) Monika kaufte Milch.

(20.b) Monika kaufte eine Glühbirne.

Unter diesem Blickwinkel sind sie nahezu äquivalent mit entsprechenden unbeschränkten $\mathrm{Ne}$ gationen, schließen allerdings auch das NichtZutreffen unendlich vieler anderer Sachverhaltsbeschreibungen ein, wie etwa:

(18.c) Monika kaufte viele Brötchen.

(19.c) Monika kaufte zwei Liter Milch.

(20.c) Monika kaufte zehn Glühbirnen.

Nullquantifikation reicht also in solchen Fällen weiter, als unbeschränkte Negation reichen würde. Der sich daraus ergebende Unterschied ist aber irrelevant, solange von variablen Gegenständen die Rede ist. Für eine zusätzliche Variante der unbeschränkten Negation mit nicht besteht hier also offenbar kein Bedarf. Es ist ökonomisch, Negation mit nicht für das Sprechen über fixierte Gegenstände zu reservieren. In der Tat werden Sätze wie die folgenden stets entsprechend verstanden:

(18.d) Monika kaufte Brötchen nicht. $(\rightarrow$ bestimmte Brötchen)

(19.d) Monika kaufte Milch nicht. $(\rightarrow$ bestimmte Milch)

(20.d) Monika kaufte eine Glühbirne nicht. ( $\rightarrow$ eine bestimmte Glühbirne)

Andererseits kann man Sätze wie (18.a) bis (20.a) auch so interpretieren, daß sie angeben, was in einer bestimmten Position nicht vorhanden ist, nicht aber das, was dort vorhanden ist. Sie lassen sich dann etwa folgendermaßen vervollständigen:

(18.e) Monika kaufte keine Brőtchen, aber Tomaten.

(19.e) Monika kaufte keine Milch, aber Bier.

(20.e) Monika kaufte keine Glühbirne, aber eine Kerze.

Unter diesem Blickwinkel ähnelt Nullquantifikation mit kein- der zugespitzt replaziven Negation der Gegenstandskategorie mit nicht:

(18.f) Monika kaufte nicht /BRÖTchen, sondern ToMA \ten.

In der Tat ist in diesem Bereich Alternation von kein- und nicht zu beobachten:

(18.g) Monika kaufte keine / BROTchen, sondern ToMA \ten.,

und zwar nicht nur bei variablen Gegenständen, sondern auch bei einzuführenden fixierten $\mathrm{Ge}$ genständen (Variante ii), also immer dann, wenn Identitätsinformation nicht kodiert wird.

Bei einzuführenden fixierten Gegenständen fungiert kein-als Indefinitartikel:

(22.a) Ich habe NICHT einen / MANN, sondern einen HUND \gesehen.

(22.b) Ich habe keinen /MANN, sondern einen HUND \gesehen.

Wo Alternation möglich ist, wird kein- in Artikelposition, nicht dagegen in Distanzposition bei Topikalisierung bevorzugt:

(23.a) Sandra mag keine Milch.

(23.b) Milch mag Sandra nicht.

(24.a) Tobias hat uns kein gutes Restaurant empfohlen.

(24.b) Ein gutes Restaurant hat Tobias uns nicht empfohlen.

Umgangssprachlich findet man daneben aber auch kein- in Distanzposition:

(25) Breitmaulfrösche gibt's hier keine.

Bei eingeführten fixierten Gegenständen ist die Verwendung von kein- ausgeschlossen. In solchen Fällen muß nicht verwendet werden:

(26.a) *Die keine Tochter des Müllers war fleißig.

(26.b) Die Tochter des Müllers war nicht fleißig.

Eine besonders interessante Arbeitsteilung von kein- und nicht ist abschließend im Bereich der Kodierung von Quantitätsinformation zu beobachten. Während man zur gewöhnlichen Nullquantifikation kein- verwendet, kann man zur emphatischen Nullquantifikation von Individuen auch nicht ein- im Sinne von ,auch nicht ein-" /noch nicht einmal ein-" benutzen:

(27.a) Ich habe KEInen Fehler gefunden.

(27.b) Ich habe nicht EInen Fehler gefunden. 
Umgekehrt dient nicht zur zugespitzt replaziven Negation der Gegenstandsquantität. Hier kann man vor einem eine Mehrzahl bezeichnenden Zahlwort auch kein-verwenden, und zwar wiederum in der emphatischen Lesart „, noch nicht einmal" / "sogar weniger als":

(28.a) Tobias hat nicht / ZWEI, sondern nur EI \nen Tag gebraucht.

(28.b) Tobias hat /KEIne zwei TA \ge gebraucht.

4. Die unterschiedlichen Aufgaben von nicht und $k e i n$ - lassen sich demnach folgendermaßen gegenüberstellen:

- Nicht ist ein Negationselement, das erstens zur unbeschränkten Negation von Sachverhaltsbeschreibungen mit fixierten Gegenständen und zweitens zur zugespitzt replaziven Negation von Beschreibungsbestandteilen aller Art verwendet werden kann. Im Bereich der Quantitätsinformation dient nicht ferner in Verbindung mit ein zur emphatischen Nullquantifikation von Individuen.

- Kein- ist ein Nullquantor. Beschreibungen von Sachverhalten mit nullquantifizierten Gegenständen können erstens als Äquivalente unbeschränkt negierter Beschreibungen von Sachverhalten mit quantifizierten variablen Gegenständen (Variante i) und zweitens, wenn explizite Identitätsinformation fehlt (Varianten i und ii), auch als Äquivalente zugespitzt replaziver Negationen der Gegenstandskategorie interpretiert werden. Im Bereich der Quantitätsinformation dient kein-ferner in Verbindung mit Zahlwörtern der Mehrzahl als emphatisches Element zur zugespitzt replaziven Negation.

Das Schema veranschaulicht die Verhältnisse im Überblick:

\begin{tabular}{|c|c|c|c|}
\hline Wissen des & variable Gegenstände & \multicolumn{2}{|l|}{ fixierte Gegenstände } \\
\hline $\begin{array}{l}\text { kommunikativer } \\
\text { Status }\end{array}$ & & einzuführen & eingeführt \\
\hline Kodierung & $\begin{array}{l}\text { (Quantor +) Deskriptor }+ \\
\text { Numerus }\end{array}$ & $\begin{array}{l}\text { (Indefinitartikel +) De- } \\
\text { skriptor + Numerus }\end{array}$ & $\begin{array}{l}\text { Determinator (+ Quan- } \\
\text { tor)(+ Deskriptor) + } \\
\text { Numerus }\end{array}$ \\
\hline & Variante i & Variante ii & Variante iii \\
\hline kein & $\begin{array}{l}\text { Nullquantifikation } \\
\text { unbeschränkte Negation } \\
\text { von Sachverhaltsbe- } \\
\text { schreibungen } \\
\text { replazive Negation der } \\
\text { Gegenstandskategorie } \\
\text { replazive Negation der } \\
\text { Gegenstandsquantität in } \\
\text { Verbindung mit Zahl- } \\
\text { wörtern der Mehrzah! } \\
\text { (emphatisch) }\end{array}$ & $\begin{array}{l}\text { replazive Negation der } \\
\text { Gegenstandskategorie }\end{array}$ & \\
\hline nicht & $\begin{array}{l}\text { Nullquantifikation von } \\
\text { Individuen in Verbin- } \\
\text { dung mit Zahlwörtern } \\
\text { der Einzahl (emphatisch) }\end{array}$ & $\begin{array}{l}\text { unbeschränkte Negation } \\
\text { von Sachverhaltsbe- } \\
\text { schreibungen } \\
\text { zugespitzt replazive Ne- } \\
\text { gation aller Art }\end{array}$ & $\begin{array}{l}\text { unbeschränkte Negation } \\
\text { von Sachverhaltsbe- } \\
\text { schreibungen } \\
\text { zugespitzt replazive Ne- } \\
\text { gation aller Art }\end{array}$ \\
\hline
\end{tabular}




\section{Anmerkungen}

I Vgl. z. B. G. Helbig/J. Buscha, Deutsche Grammatik. Ein Handbuch für den Ausländerunterricht, Leipzig ${ }^{9} 1986$, S. 390;

U. Engel, Deutsche Grammatik, Heidelberg 1988, S. 786 f.

2 Vgl. G. Helbig/J. Buscha, ebenda, S. 390 ff.;

U. Engel, ebenda, S. $786 \mathrm{ff}$.;

E. Hentschel / H. Weydt, Handbuch der deutschen Grammatik, Berlin 1990, S.294f.

3 Vgl. H. Blühdorn, Funktionale Zeichentheorie und deskriptive Linguistik. Ein Entwurf am Beispiel des Gegenwartsdeutschen, Erlangen 1993, S. $15 \mathrm{f}$.

$4 \mathrm{Vgl}$. H. Weinrich, Textgrammatik der deutschen Sprache, Mannheim 1993, S. 864.
5 Die Großbuchstaben zeigen die Lage des Satzakzentes. Mit den Schrägstrichen vor bzw, nach den Akzentsilben wird zwischen steigendem und fallendem Akzent unterschieden.

6 Vgl. J. Jacobs, Negation, in: A. von Stechow/D. Wunderlich (Hg.), Semantik. Ein internationales Handbuch der zeitgenössischen Forschung, Berlin 1991, S. $585 \mathrm{ff}$.

7 So etwa P. Eisenberg, Grundriß der deutschen Grammatik, Stuttgart ${ }^{2} 1989$, S. 167.

$8 \mathrm{Vgl}$. dazu u.a. H.Vater, Der unbestimmte Artikel als Quantor, in: W. Welte ( $\mathrm{Hg}$.), Sprachtheorie und angewandte Linguistik. Festschrift für Alfred Wollmann, Tübingen 1982, S. $70 \mathrm{f}$. 